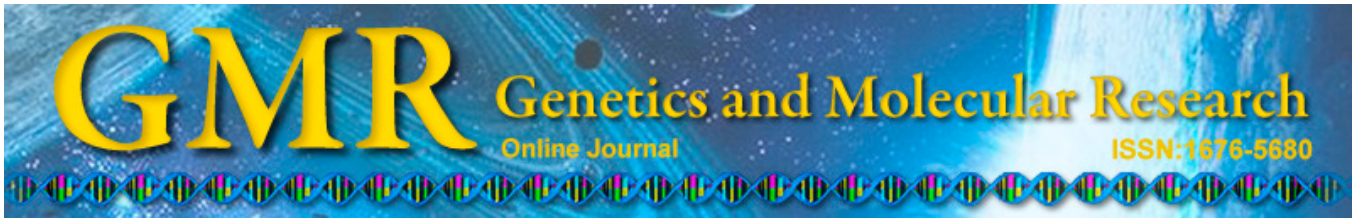

\title{
Role of the CKIP1 gene in proliferation and apoptosis of the human lung cancer cell line H1299
}

\author{
G.M. Chen ${ }^{1}$, R.F. Ding ${ }^{2}$, Y.D. Tan², X.B. Pan' ${ }^{2}$ G.M. Jiang ${ }^{2}$, J.F. He ${ }^{3}$, \\ S.H. Lin' ${ }^{2}$ C. $\mathrm{Liu}^{2}$ and Y. Jia ${ }^{2}$ \\ ${ }^{1}$ Surgical Department, Dongguan People's Hospital, Dongguan City, China \\ ${ }^{2}$ Department of Medical Oncology, Dongguan People's Hospital, \\ Dongguan City, China \\ ${ }^{3}$ Pathology Department, Dongguan People's Hospital, Dongguan City, China \\ Corresponding author: Y. Jia \\ E-mail: dgryjy@hotmail.com
}

Genet. Mol. Res. 14 (2): 4005-4014 (2015)

Received June 13, 2014

Accepted October 7, 2014

Published April 27, 2015

DOI http://dx.doi.org/10.4238/2015.April.27.15

\begin{abstract}
Casein kinase 2 interacting protein 1 (CKIP1) is a specific interacting protein of the casein kinase 2 (CK2) $\alpha$ subunit, and, by binding $\mathrm{CK} 2$ and other proteins, functions as an adaptor to regulate a series of cellular functions. Previous studies suggested that CKIP1 might play an important role in regulating oncogenic activities. However, few studies examining the function of CKIP1 in cancer cells have been performed. The present study aimed to investigate the role of CKIP1 in lung cancer. CKIP1 mRNA expression was detected in 5 human lung cancer cell lines (H-125, H1299, LTEP-A-2, SPC-A-1, and NCL-H446) by semi-quantitative RT-PCR, and in 10 noncancerous lung tissues and 30 non-small lung cancer tissues by real-time quantitative PCR. A lentivirus-mediated small interfering RNA (siRNA) was used to knock down CKIP1 expression in the H1299 cell line. To elucidate the impact of CKIP1 downregulation on H1299 cells, cell proliferation, DNA synthesis, and cell cycle distribution and apoptosis
\end{abstract}


were measured by high content screening assay, BrdU incorporation, and flow cytometric analyses, respectively. CKIP1 mRNA was highly expressed both in $\mathrm{H} 1299$ cells and lung cancer tissues. We found that downregulation of CKIP1 resulted in suppression of proliferation and colony-forming ability of H1299 cells, and led to S phase cell cycle arrest and G2 phase promotion, as well as a significant enhancement of H1299 cell apoptosis. Our study indicated that high expression levels of CKIP1 were associated with the development of lung cancer, and that CKIPl knockdown may block tumor cell growth mainly by promoting cell apoptosis.

Key words: Casein kinase 2 interacting protein 1; Lung cancer; MicroRNA; Lentivirus

\section{INTRODUCTION}

Lung cancer is the most common type of cancer, and is the leading cause of cancerrelated deaths worldwide. More than a million new patients will suffer from lung cancer each year (Toh, 2009). In China, data from the national death survey and cancer registration on the mainland show that lung cancer is the leading cause of death in most of cities (Chen, 2009). Deaths from this typically fatal disease have increased nearly five-fold since the 1970s. Therefore, it is vital to find the key factors underlying lung cancer and take effective measures to control the rising carcinoma incidence.

Epidemiologic studies have reported that most etiological factors underlying lung cancer come from the environment, including cigarette smoking and occupational exposure to air pollution (Spitz et al., 2008). However, only a small portion of individuals who have been exposed to hazardous environmental factors develop lung cancer. This suggests that genetic factors may predispose individuals to develop lung cancer (Amos et al., 1992). Recently, a meta-analysis including results from 41 studies revealed that a family history of lung cancer is a risk factor for lung cancer (Lissowska et al., 2010). This indicates that genetic factors also play an important role in the development of human lung cancer.

The casein kinase 2 interacting protein 1 (CKIPl) gene encodes a protein of $\sim 46 \mathrm{kDa}$, with a pleckstrin homology $(\mathrm{PH})$ domain at the $\mathrm{N}$-terminus and a putative leucine zipper (LZ) motif at the C-terminus, as well as five praline-rich motifs throughout the protein. CKIP1 was originally identified in a yeast two-hybrid screen as an interacting protein of casein kinase 2 (CK2) at specific cellular locations (Bosc et al., 2000). A previous study demonstrated that the pleckstrin homology domain of CKIP1 was required for interactions with CK2 and for the recruitment of CK2 to the plasma membrane (Olsten et al., 2004). CKIP1 also functions as an adaptor, by binding CK2 and other proteins, to regulate a series of cellular functions (Lawrie et al., 2008). CKIP1 is implicated in the regulation of cell apoptosis, through repression of the transcriptional activity of AP1 (Zhang et al., 2005). Large-scale investigations have suggested that the cell context-dependent localization of CKIP1 might play an important part in the activity and subcellular localization of both CK1 and Akt, and regulate their oncogenic activities (Hennessy et al., 2005; Lu et al., 2008). Recently, a sequence homology and bioinformatic analysis revealed the potential tumor suppression ability of CKIP1 (Koskimaki et al., 2012). However, few studies have performed experimental verification of the potential function of CKIP1 in cancer cells. 
In this study, we investigated the relationship between CKIP1 and human lung cancer development, and the effects of CKIP1 downregulation in the human lung cancer H1299 cell line.

\section{MATERIAL AND METHODS}

\section{Cell culture}

The human non-small cell lung cancer cell lines used in this study were H-125, H1299, LTEP-A-2, SPC-A-1, and NCL-H446, which were obtained from the American Type Culture Collection (ATCC, Rockville, MD, USA). Human renal epithelial 293T cells were purchased from the Cell Bank Type Culture Collection of the Chinese Academy of Sciences (CBTCCCAS, Shanghai, China). All cells were grown in DMEM (GIBCO, Carlsbad, CA, USA) with $10 \%$ fetal bovine serum (FBS; GIBCO) at $37^{\circ} \mathrm{C}$ in a humidified atmosphere of $5 \% \mathrm{CO}_{2}$.

\section{Semi-quantitative reverse transcription polymerase chain reaction (RT-PCR)}

Total RNA from cell lines H-125, H1299, LTEP-A-2, SPC-A-1, and NCL-H446 was extracted using TRIzol reagent (Invitrogen, Carlsbad, CA, USA) according to the manufacturer protocol. In brief, $2 \mu \mathrm{g}$ total RNA from each sample was reverse transcribed to single-stranded cDNA (Invitrogen, Carlsbad, CA, USA). We used $1 \mu \mathrm{L}$ cDNA as the template for PCR. The primer sequences for PCR amplification of the CKIP1 gene were 5'-ATCACCCGAGCCAAGAACC-3' and 5'-GGAAGCCACAGCCATTAGG-3'. $G A P D H$ was applied as an internal control. The primer sequences for GAPDH were 5'-TGACTTCAACAGCGACACCCA-3' and 5'-CACCCTGTTGCTGTAGCCAAA-3'.

The semi-quantitative RT-PCR comprised an initial denaturation at $95^{\circ} \mathrm{C}$ for $15 \mathrm{~s}$, then 22 cycles at $95^{\circ} \mathrm{C}$ for $5 \mathrm{~s}$ and $60^{\circ} \mathrm{C}$ for $30 \mathrm{~s}$. PCR products were run on a $2 \%$ agarose gel.

\section{Real-time quantitative PCR}

For real-time quantitative PCR (TAKARA, TP800), tumor samples were obtained from 30 patients who were diagnosed with non-small lung cancer and enrolled at Dongguan People's Hospital. Ten noncancerous lung tissues from corresponding adjacent tissues of the 30 tumor tissues were used as control group. Before the study, written informed consent was obtained from all participants. This study was approved by the Zhongshan Hospital of Hubei Ethics Committee.

Total RNA was isolated from samples using TRIzol reagent according to manufacturer protocols. cDNA was prepared from 2-5 $\mu$ g total RNA using Superscript II reverse transcriptase (Invitrogen) and random hexamer primers. We used $1 \mu \mathrm{L}$ cDNA for real-time PCR to detect CKIP1 using a SYBR Green Mixture (TaKaRa Bio., Otsu, Japan) according to the manufacturer protocol. Primers were as used in semi-quantitative PCR. Data were analyzed using the GraphPad PRISM4.0 Software (GraphPad Software Inc., San Diego CA, USA). Results were presented as CT values, defined as the threshold PCR cycle number at which an amplified product was first detected. The average CT was calculated for both CKIPI and $G A P D H$, and $\triangle \mathrm{CT}$ was determined as the mean of the triplicate CT values for CKIPI minus the mean of the triplicate CT values for $G A P D H$. The $2^{-\Delta \Delta \mathrm{Ct}}$ method was used to analyze the relative changes in gene expression. 


\section{Construction of CKIP1 siRNA lentivirus}

siRNA was designed to target the CKIPI ORF sequence (GenBank No. NM_016274). The sequence of the CKIP1 siRNA was 5'-GAGCTGAGAGACCTGTACAGA-3'. A non-targeting sequence was used as a lentivirus negative control and was purchased from Shanghai GeneChem, Co. Ltd. (Shanghai, China). The sequences were cloned into the pGCSIL-GFP vector (GeneChem). Lentiviral-based siRNA-expressing vectors were confirmed by DNA sequencing. Human renal epithelial 293 T cells were transfected with CKIP1 siRNA lentivirus or control lentivirus. The interference efficiency of the template was detected by Western blot analysis. H1299 cells were transfected with CKIP1 siRNA or control lentivirus. After 3 days of infection, GFP expression was observed by fluorescent microscopy. After 5 days of infection, cells were harvested to determine knockdown efficiency by real-time quantitative PCR.

\section{Western blot}

Two hundred and ninety three $\mathrm{T}$ cells were collected after $36 \mathrm{~h}$ transfection and total protein was isolated from the cells and quantitated by BSA. Protein $(20 \mu \mathrm{g})$ was loaded onto a $10 \%$ SDS-PAGE gel and transferred to PVDF membranes (Millipore). Proteins were detected by their respective antibodies (Mouse Anti-GFP: Santa-Cruz, sc-9996, 1:2000; Mouse antiGAPDH: Santa-Cruz, sc-32233, 1:5000) using an ECL kit (Amersham BioSciences, Woburn, MA, USA) and exposed to X-ray film. The protein level of GAPDH was used as a control and detected by anti-GAPDH (Santa Cruz Biotechnology, Santa Cruz, CA, USA).

\section{Cell proliferation assay}

Cell growth was measured by counting the viable cell number with Cellomics Array$\mathrm{Scan}^{\mathrm{TM}}$ VTI HCS Reader (Thermo Scientific, Waltham, MA, USA) according to manufacturer instructions. In brief, H1299 cells at 10 days post-transfection with CKIP1 siRNA or control lentivirus were seeded at 2000 cells per well on 96 -well plates, and then incubated at $37^{\circ} \mathrm{C}$ with $5 \% \mathrm{CO}_{2}$. The viable cells transfected with lentivirus carrying GFP were detected with the Cellomics machine. The cell number was counted each day after plating for five successive days.

\section{BrdU incorporation assay}

DNA synthesis in proliferating cells was determined by BrdU incorporation. Cells were spread onto 96-well plates and incubated for 24 or $96 \mathrm{~h}$. We added $10 \mu \mathrm{L} 1 \mathrm{X}$ 5-bromodeoxyuridine (BrdU) reagent (BrdU kit, Roche, Cat. No. 11647229001) from 2 to 24 h; then $100 \mu \mathrm{L}$ Fixing Solution was added to the cells for $30 \mathrm{~min}$. The cells were washed with Wash Buffer and incubated for 60 min with $50 \mu \mathrm{L} 1 \mathrm{X}$ BrdU antibody. After adding $50 \mu \mathrm{L} 1 \mathrm{X}$ Goatanti-Mouse IgG, $50 \mu \mathrm{L}$ TMB substrate solution was added. Following $30 \mathrm{~min}$ incubation, the stop solution was added. The OD was measured at $450 \mathrm{~nm}$ using a plate reader.

\section{Fluorescence-activated cell sorting analysis}

The cell cycle distribution was analyzed by flow cytometry. In brief, 1 x $10^{6}$ cells were seeded on six-well plates in triplicate and incubated at $37^{\circ} \mathrm{C}$ for 5 days. The cells were col- 
lected, washed with PBS, and fixed with 70\% cold ethanol. The cells were then treated with $100 \mu \mathrm{g} / \mathrm{mL}$ DNase-free RNase. Propidium iodide (50 $\mu \mathrm{g} / \mathrm{mL}$; Sigma P4170, St. Louis, MO, USA) was added directly to the cell suspension and a total of 10,000 fixed cells were analyzed for the cell cycle phase by FACScan (Becton-Dickinson, Franklin Lakes, NJ, USA).

Apoptotic cells were detected with the Annexin V-APC Apoptosis Detection Kit (eBioscience, Inc., San Diego, CA, USA). In brief, $5 \times 10^{5}$ cells were collected by centrifugation. The cells were resuspended in $500 \mu \mathrm{L} 1 \mathrm{X}$ binding buffer. A total of $5 \mu \mathrm{L}$ Annexin V-APC and $5 \mu \mathrm{L}$ PI were added and incubated at RT for 5 min in the dark. Finally, cells were analyzed by FACScan.

\section{Formation of colonies}

A total of $800 \mathrm{H} 1299$ cells were seeded into six-well plates. The medium was changed every three days. After two weeks of culture, the cells were washed with PBS. Freshly diluted Giemsa stain (Chemicon ECM550, Pittsburgh, PA, USA) was added and allowed to react for at least $30 \mathrm{~min}$. After being rinsed in distilled water, the cells were differentiated with $0.5 \%$ aqueous acetic acid within $30 \mathrm{~s}$ and were dehydrated rapidly. Colonies with more than 50 cells were counted under fluorescence microscopy.

\section{Statistical analysis}

One-way ANOVA and Student $t$-test were used for raw data analysis. Statistical analysis was performed using the SPSS17.0 software package (SPSS, Chicago, IL, USA). All values in the text and figures are reported as means $\pm \mathrm{SD}$. $\mathrm{P}<0.05$ was considered to be statistically significant.

\section{RESULTS}

\section{Measurement of CKIP1 mRNA in lung cancer cell lines and human tissues}

The expression of CKIP1 in cell lines H-125, H1299, LTEP-A-2, SPC-A-1, and NCL-H446 was detected by semi-quantitative RT-PCR. CKIP1 mRNA was expressed in all cell lines examined (Figure 1A). In order to confirm the correlation between lung cancer and CKIP1 mRNA expression, CKIP1 gene differential expression on noncancerous lung tissue and non-small lung cancer tissue was observed by real-time quantitative PCR (Figure 1B). A higher expression level of CKIP1 was detected in 30 lung cancer samples compared with that in noncancerous lung cancer tissues $(\mathrm{P}=0.036)$.

\section{CKIP1 siRNA lentiviral suppression of CKIP1 expression}

To detect the knockdown efficiency of the siRNA target sequence, CKIP1 protein level was detected by Western blot in 293T cells. As shown in Figure 2A, the expression of CKIP1 protein was significantly suppressed after lentiviral transfection. CKIP1 siRNA or control lentivirus was transfected into H1299 cells and after 5 days, CKIP1 mRNA levels were detected by real-time quantitative PCR. As shown in Figure 2B, CKIP1 siRNA lentivirus transfected cultures had significantly lower levels of CKIP1 mRNA compared to cultures transfected with control lentivirus. 
A

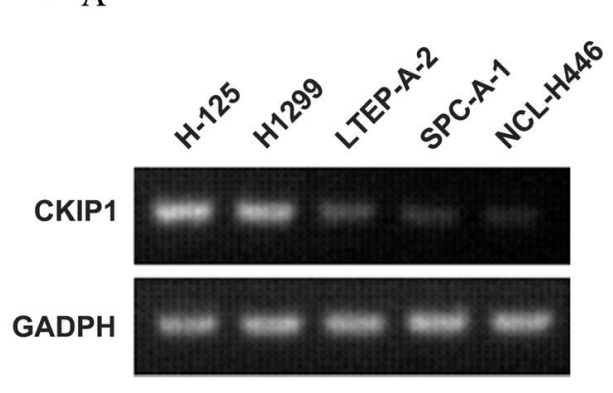



Figure 1. Expression of CKIP1 mRNA in lung cancer cell lines and tissues. A. CKIP1 mRNA was measured by semi-quantitative RT-PCR in five lung cancer cell lines (H-125, H1299, LTEP-A-2, SPC-A-1, and NCL-H446). B. CKIPI mRNA differential expression was detected by real-time quantitative PCR in tissues. The $2^{-\Delta \Delta C t}$ method was used to analyze the relative changes in gene expression. $* * \mathrm{P}<0.05$.
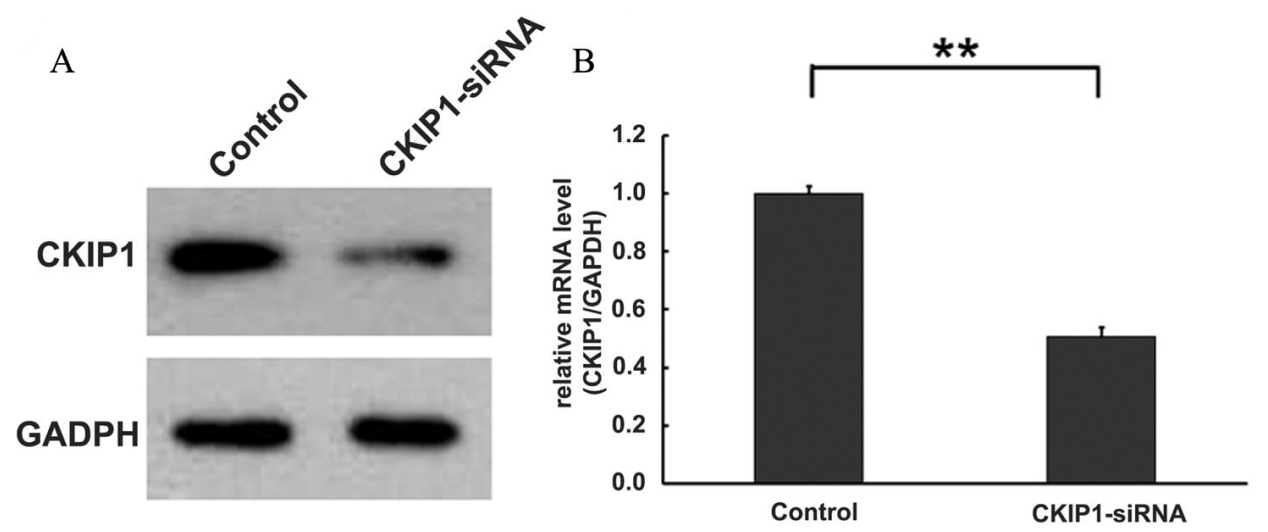

Figure 2. CKIP1 knockdown efficiency. A. CKIP1 protein in 293 T cells measured by Western blot. B. CKIP1 mRNA levels in H1299 cells at the 5th day following transfection with CKIP1 siRNA or control lentivirus, detected by real-time quantitative PCR. $* * \mathrm{P}<0.01$.

\section{Downregulation of CKIP1 in the human malignant cell line H1299 inhibits cell growth}

In order to explore the function of CKIP1 in lung cancer, we first examined cell growth in CKIP1 siRNA or control lentivirus infected H1299 cells by high-content screening (HCS) and BrdU incorporation. After 5 days of culture, the number of H1299 cells transfected with normal control lentivirus increased more than ten-fold, while the CKIPI siRNA infected cells only increased four-fold (Figure 3A-C). Downregulation of CKIP1 decreased the total number of cells. We further found that compared with the control group, the colony numbers of H1299 cells displayed a consistent reduction when cells were transfected with CKIP1 siRNA lentivirus (Figure 4A and B). Furthermore, the levels of DNA synthesis 24 and $96 \mathrm{~h}$ after transfection with CKIP1 siRNA lentivirus were decreased (Figure 4C). These results indicated that suppression of CKIP1 expression inhibited proliferation, DNA synthesis and colony formation of H1299 cells. 


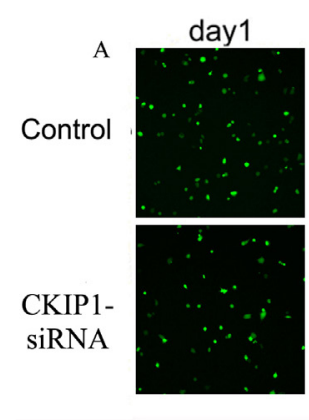

B



day2
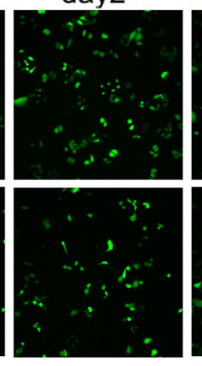

time


day5

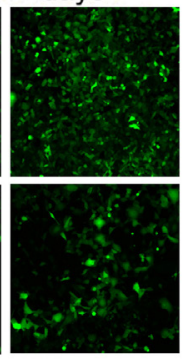

$\mathrm{C}$

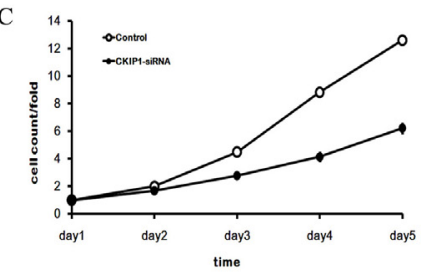

Figure 3. Effect of CKIP1 downregulation on H1299 cell growth. H1299 cells transfected with CKIP1 siRNA or control lentivirus were seeded into 96 -well plates for 5 days. A. High content cell imaging assays were applied to acquire raw images of cell growth. B. Cell growth was assayed every day for 5 days. C. Cell growth rate was monitored on each day by the HCM assay.
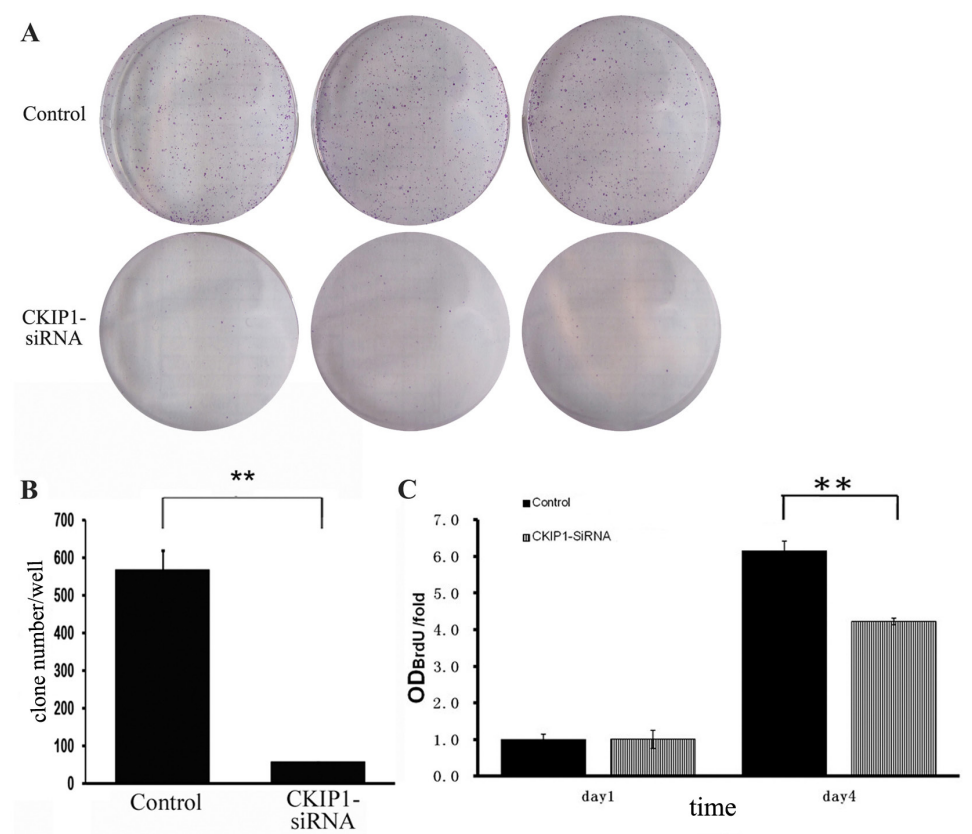

Figure 4. CKIP1 downregulation in H1299 cells decreased colonies number and suppressed DNA synthesis. A. Colony formation of H1299 cells in a culture plate. CKIP1 siRNA or control lentivirus transfected cells ( 800 cells per well) on six-well plates were cultured for 2 weeks and stained with Giemsa. B. Quantitative analysis of colony formation. C. BrdU incorporation was used to determine the DNA synthesis on the first and fourth days after transfection with lentivirus. Data is shown with blank control. Mean results $\pm \mathrm{SD}$ of a representative experiment performed in triplicate. ${ }^{* *} \mathrm{P}<0.01$. 


\section{CKIP1 reduction affects $\mathrm{H1299}$ cell cycle progression}

To elucidate the impact of downregulated CKIP1 expression on H1299 cell cycling, FACS analysis was performed at $96 \mathrm{~h}$ after lentiviral transfection. As shown in Figure 5A, in the control group, the cells in $\mathrm{G} 0 / \mathrm{G} 1, \mathrm{~S}$, and $\mathrm{G} 2 / \mathrm{M}$ phases accounted for $62.7,28.5$, and $8.7 \%$ of the population, respectively. However, in the experimental group, the cells in G0/G1, S, and G2/M phases accounted for 65, 22.3, and 12.7\%, respectively. As shown in Figure 5B, the CKIP1 siRNA transfected group displayed a significant decrease in the percentage of cells in $\mathrm{S}$ phase $(\mathrm{P}=0.019)$. A significant increase of cells in $\mathrm{G} 2$ phase was observed in the CKIP1 siRNA group compared to the control group $(\mathrm{P}=0.027)$. This suggested that cells were arrested in S phase and vitalized in G2 phase when CKIP1 expression was reduced.

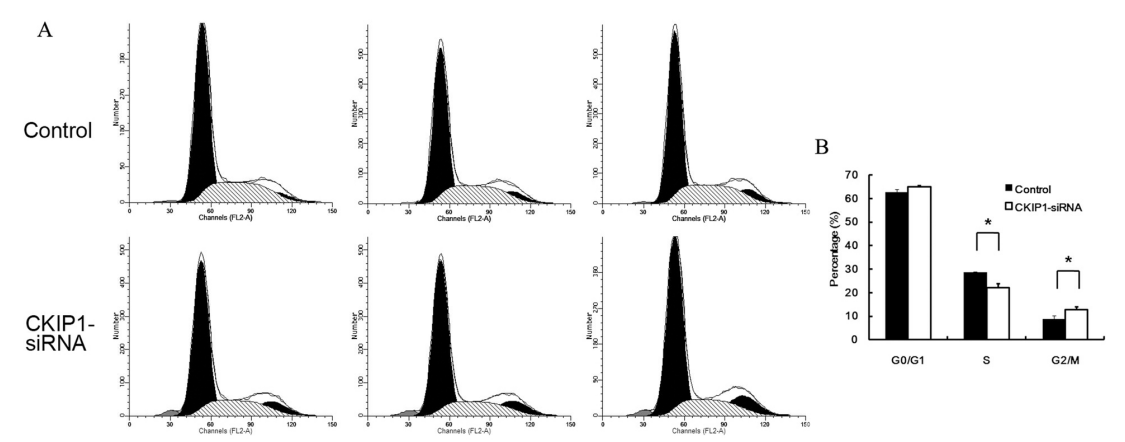

Figure 5. CKIP1 downregulation in H1299 cells arrested the cell cycle. A. The cell cycle of H1299 cells was analyzed by flow cytometry. B. Compared with the control group, CKIP1 siRNA transfected cultures showed a significant difference in the percentages of cells in $\mathrm{S}$ and G2 phases. ${ }^{*} \mathrm{P}<0.05$.

\section{Effect of CKIP1 downregulation on apoptosis in H1299 cells}

To determine if downregulating CKIP1 affects cell survival, the level of cell apoptosis was detected by a flow cytometry assay. Cell apoptosis was determined by Annexin-V staining (Figure 6A). As shown in Figure 6B, the level of cell apoptosis was significantly increased in the CKIP1 siRNA transfected group compared to the control group $(\mathrm{P}=0.0059)$. These results indicated that CKIP1 expression was significantly associated with lung cancer cell apoptosis.

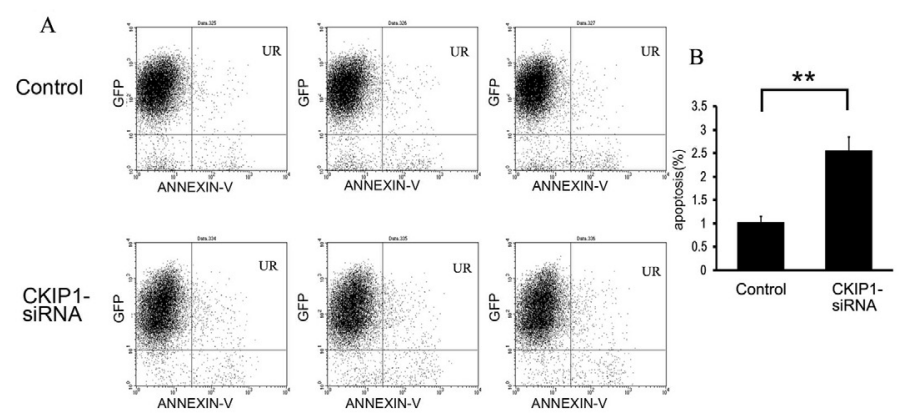

Figure 6. Effect of CKIP1 downregulation on apoptosis in H1299 cells. A. Cell death was detected by Annexin V staining and flow cytometry. UR $=$ apoptosis cells. B. Percentage of cell apoptosis in the CKIP1 siRNA transfected and control groups. ${ }^{*} \mathrm{P}<0.01$. 


\section{DISCUSSION}

CKIP1 has been reported to regulate several important proteins that control cell survival, such as CK2, Akt, IFP53 and Smurf1, all of which have also been associated with human cancers (Hennessy et al., 2005; Lu et al., 2008). In addition, CKIP1 negatively regulates several important pathways, such as TGF- $\beta$ /BMP and PI3K/Akt signaling, which participate in cancer stem cell regulation (Martelli et al., 2011; Ibanez et al., 2012). As discussed above, CKIP1 might play an important part in the development of human cancers. In the present study, we tried to explore the function of CKIP1 in human lung cancer.

It has been reported that CKIP1 was expressed abundantly in normal tissues, but was expressed only at very low levels in most cancer cell lines and some cancer tissues (Tokuda et al., 2007; Zhang et al., 2007). However, we found that CKIP1 expression levels in lung cancer tissues were higher than those in the normal tissues. Furthermore, using GAPDH as an internal reference, our semi-quantitative PCR results showed that CKIP1 had high expression in the H1299 human lung cancer cell line. These results indicated that CKIP1 might have specific functions in lung cancer.

In order to assess CKIP1 function in lung cancer cell lines, we transfected H1299 cells with CKIP1 siRNA or control lentivirus. Compared to controls, CKIP1 siRNA treated cells showed decreased proliferation and increased apoptosis. In addition, monitoring the checkpoint of cell cycle of the two group cells revealed a decrease in the proportion of cells in $S$ phase and an increase those in G2 phase. Together, these results suggested that CKIP1 promoted H1299 cell growth. It is well known that the S phase is the period of DNA synthesis, which is a critical point for cells to commit to proliferation or undergo growth arrest (Sherr, 2000). Regulation of the $S$ phase transition would affect the rate of cell growth. Our data suggested that CKIP1 enhanced the proportion of cells in S phase. This confirmed our conclusion that CKIP-1 increased lung cancer H1299 cell growth.

However, recent reports by Safi et al. (2004) and Tokuda et al. (2007) clearly demonstrated that CKIP1 could inhibit cell growth by repressing Akt phosphorylation and kinase activity. Zhang et al. (2005) also had shown that CKIP1 could promote apoptosis via caspase3 -dependent cleavage and translocation. It was determined that the sub-cellular localization of CKIP1 influenced its function, regulating the balance between cell growth and apoptosis by interacting with specific signals. Therefore, we hypothesized that CKIP1 might have still different localization in the human lung cancer H1299 cell lines, causing inverse functions of CKIP1 in controlling cell proliferation and apoptosis. Although we have demonstrated a correlation between CKIP1 expression and cell proliferation in the lung cancer cell line H1299, its function in lung cancer is still unclear. Further studies should focus on elucidating the molecular mechanism and signaling pathways controlling survival and activity of lung cancer.

\section{REFERENCES}

Amos CI, Caporaso NE and Weston A (1992). Host factors in lung cancer risk: a review of interdisciplinary studies. Cancer Epidemiol. Biomarkers Prev. 1: 505-513.

Bosc DG, Graham KC, Saulnier RB, Zhang C, et al. (2000). Identification and characterization of CKIP-1, a novel pleckstrin homology domain-containing protein that interacts with protein kinase CK2. J. Biol. Chem. 275: 14295-14306.

Chen WQ (2009). Estimation of cancer incidence and mortality in China in 2004-2005. Zhonghua Zhong Liu Za Zhi. 31: 664-668. 
Hennessy BT, Smith DL, Ram PT, Lu Y, et al. (2005). Exploiting the PI3K/AKT pathway for cancer drug discovery. Nat. Rev. Drug Discov. 4: 988-1004.

Ibanez E, Agliano A, Prior C, Nguewa P, et al. (2012). The quinoline imidoselenocarbamate EI201 blocks the AKT/mTOR pathway and targets cancer stem cells leading to a strong antitumor activity. Curr. Med. Chem. 19: 3031-3043.

Koskimaki JE, Rosca EV, Rivera CG, Lee E, et al. (2012). Serpin-derived peptides are antiangiogenic and suppress breast tumor xenograft growth. Transl. Oncol. 5: 92-97.

Lawrie CH, Gal S, Dunlop HM, Pushkaran B, et al. (2008). Detection of elevated levels of tumour-associated microRNAs in serum of patients with diffuse large B-cell lymphoma. Br. J. Haematol. 141: 672-675.

Lissowska J, Foretova L, Dabek J, Zaridze D, et al. (2010). Family history and lung cancer risk: international multicentre case-control study in Eastern and Central Europe and meta-analyses. Cancer Causes Control. 21: 1091-1104.

Lu K, Yin X, Weng T, Xi S, et al. (2008). Targeting WW domains linker of HECT-type ubiquitin ligase Smurf1 for activation by CKIP-1. Nat. Cell Biol. 10: 994-1002.

Martelli AM, Evangelisti C, Follo MY, Ramazzotti G, et al. (2011). Targeting the phosphatidylinositol 3-kinase/Akt/ mammalian target of rapamycin signaling network in cancer stem cells. Curr. Med. Chem. 18: 2715-2726.

Olsten ME, Canton DA, Zhang C, Walton PA, et al. (2004). The Pleckstrin homology domain of CK2 interacting protein-1 is required for interactions and recruitment of protein kinase CK2 to the plasma membrane. J. Biol. Chem. 279: 42114-42127.

Safi A, Vandromme M, Caussanel S, Valdacci L, et al. (2004). Role for the pleckstrin homology domain-containing protein CKIP-1 in phosphatidylinositol 3-kinase-regulated muscle differentiation. Mol. Cell Biol. 24: 1245-1255.

Sherr CJ (2000). The Pezcoller lecture: cancer cell cycles revisited. Cancer Res. 60: 3689-3695.

Spitz MR, Etzel CJ, Dong Q, Amos CI, et al. (2008). An expanded risk prediction model for lung cancer. Cancer Prev. Res. 1: 250-254.

Toh CK (2009). The changing epidemiology of lung cancer. Methods Mol. Biol. 472: 397-411.

Tokuda E, Fujita N, Oh-hara T, Sato S, et al. (2007). Casein kinase 2-interacting protein-1, a novel Akt pleckstrin homology domain-interacting protein, down-regulates PI3K/Akt signaling and suppresses tumor growth in vivo. Cancer Res. 67: 9666-9676.

Zhang L, Xing G, Tie Y, Tang Y, et al. (2005). Role for the pleckstrin homology domain-containing protein CKIP-1 in AP-1 regulation and apoptosis. EMBO J. 24: 766-778.

Zhang L, Tang Y, Tie Y, Tian C, et al. (2007). The PH domain containing protein CKIP-1 binds to IFP35 and Nmi and is involved in cytokine signaling. Cell Signal. 19: 932-944. 